\title{
New Technology for Microfabrication and Testing of a Thermoelectric Device for Generating Mobile Electrical Power
}

\author{
Narasimha S. Prasad ${ }^{* a}$, Patrick J. Taylor ${ }^{b}$, Sudhir B. Trivedi ${ }^{\mathrm{c}}$, Susan W. Kutcher ${ }^{\mathrm{c}}$ \\ ${ }^{a}$ NASA Langley Research Center, 5 N. Dryden St., MS 468, Hampton, VA 23681; \\ ${ }^{\mathrm{b}}$ US Army Research Laboratory, 2800 Powder Mill Road, Adelphi, MD 20783; \\ 'Brimrose Technology Corporation, 19 Loveton Circle, Baltimore, MD 21152
}

\begin{abstract}
We report the results of fabrication and testing of a thermoelectric power generation module. The module was fabricated using a new "flip-chip" module assembly technique that is scalable and modular. This technique results in a low value of contact resistivity $\left(\leq 10^{5} \Omega-\mathrm{cm}^{2}\right)$. It can be used to leverage new advances in thin-film and nanostructured materials for the fabrication of new miniature thermoelectric devices. It may also enable monolithic integration of large devices or tandem arrays of devices on flexible or curved surfaces. Under mild testing, a power of $22 \mathrm{~mW} / \mathrm{cm}^{2}$ was obtained from small $(<100 \mathrm{~K})$ temperature differences. At higher, more realistic temperature differences, $\sim 500 \mathrm{~K}$, where the efficiency of these materials greatly improves, this power density would scale to between 0.5 and $1 \mathrm{Watt} / \mathrm{cm}^{2}$. These results highlight the excellent potential for the generation and scavenging of electrical power of practical and usable magnitude for remote applications using thermoelectric power generation technologies.
\end{abstract}

Keywords: Nanostructured materials, thermoelectric power generators, flip-chip module, $\mathrm{PbTe}: \mathrm{Bi}, \mathrm{PbTe}: \mathrm{Tl}$

\section{INTRODUCTION}

Thermoelectric (TE) power generation is an increasingly important power generation technology. Significant advantages include: no moving parts, low-weight, modularity, covertness/silence, high power density, low amortized cost, and long service life with no required maintenance. However, the efficiency of TE power generation is generally considered low. Despite the low efficiency, there are many needs for electrical power that TE technologies can uniquely and successfully address. Recent advances in thermoelectric materials technology have rekindled acute interest in thermoelectric power generation .

The performance of thermoelectric power generators can be described by 2 principal metrics: (1) Thermodynamic efficiency i.e. the ratio of electrical power generated to the heat flow used for generation; and (2) Power density i.e. the electrical power generated per unit device area. These metrics depend upon the characteristics of thermoelectric materials, the device fabrication, and load driven by the generator.

Two important criteria for TE power technologies are the (power-per-unit-area) and (power-per-unit-weight) values. Power-per-unit-area values depend strongly on $\mathrm{Z}$ and $\Delta \mathrm{T}$, and commonly range in the $\sim 1 \mathrm{Watt} / \mathrm{cm}^{2}$ magnitude for reasonably large $\Delta \mathrm{T}$. These two criteria can be improved by improving the overall efficiency. The efficiency, $\Phi$, with which electrical power can be generated by TE technologies, depends on the temperature difference $\Delta \mathrm{T}$ and the $\mathrm{ZT}_{\text {avg }}$ factor [1] as given by: 


$$
\Phi=\frac{\Delta \mathrm{T}}{\mathrm{T}_{\text {hot }}}\left(\frac{\sqrt{1+\mathrm{ZT}_{\mathrm{avg}}}-1}{\sqrt{1+\mathrm{ZT}_{\mathrm{avg}}}+\mathrm{T}_{\text {cold }} / \mathrm{T}_{\text {hot }}}\right) \quad \text { where } \mathrm{Z}=\frac{\left(\alpha^{2}\right)}{\rho \kappa}
$$

$\mathrm{T}_{\mathrm{avg}}$ is simply the average of the hot and cold temperatures that define $\Delta \mathrm{T}$. So increased efficiency of power generation can be expected from a material if the thermal conductivity is decreased.

The challenge in the development of superior TE materials is in achieving high electrical conductivity, high thermoelectric power and low thermal conductivity all at the same time. Because these three material properties are all interdependent, it has proven difficult to improve one parameter of ZT without deteriorating the others. However, in the past few years some experimental evidence has been reported that indicate significant improvements in ZT. Current advancement in bulk material growth and processing techniques are enabling the practical development of improved efficiency thermoelectric devices. It has also been shown that TE performance can be improved by exploiting quantum size effects, since the transport and conversion characteristics of micro / nanostructures can differ from bulk materials significantly.

Recent developmental research has shown that the thermal conductivity of a material can be decreased by incorporating nanostructures (superlattices, nanodots, etc.) of dissimilar composition into the material [2-4]. Another approach is to introduce a high number of interfaces within a material. The best example of such interfaces is the simple grain boundary. The reduction of thermal conductivity by grain boundary scattering is well known. [5] However, the advent of technology to produce uniform nanostructured material is new. Only recently has a nanostructured bulk TE material been successfully shown [6].

\section{FABRICATION OF PbTe BASED THERMOELECTRIC POWER GENERATOR}

In this paper, we report on our study of PbTe TE material. We have previously used Bi:PbTe as the n-type material and $\mathrm{Sb} 2 \mathrm{Te} 3: \mathrm{Ag}$ as the p-type material. However, we found that $\mathrm{PbTe}$ is a more robust material compared to $\mathrm{Sb} 2 \mathrm{Te} 3: \mathrm{Ag}$, which can be flaky and hard to work with. Also, fabricating the TE power generators such that both p- and n-type elements were made of the same materials alleviates the problem of dissimilar thermal expansion at the $\mathrm{p}$ - and $\mathrm{n}$ - type element, improving the longevity and providing maintenance free power generation operation.

We began with 6n tellurium, 5n lead, 5n bismuth, and 3n thallium telluride from Alpha Aesar. Stoichiometric quantities were loaded into the ampoules and synthesized at $1000^{\circ} \mathrm{C}$. The doped PbTe crystals were grown using a 3 zone Bridgman furnace. We produced $\mathrm{n}$ type $\mathrm{PbTe}$ by doping it with $\mathrm{Bi}$ and $\mathrm{p}$-type $\mathrm{PbTe}$ by doping it with thallium. Doping levels for $\mathrm{Bi}$ were varied from 1 to $7 \times 10^{19} \mathrm{~cm}^{-3}$, and doping levels of Th varied from $5 \times 10^{19}$ to $1 \times 10^{20} \mathrm{~cm}^{-3}$. We successfully grew single crystals via the Bridgman technique using a 3-zone furnace. The p-type and n-type crystals were removed from the fused silica ampoules and cut into $3 \mathrm{~mm} \times 3 \mathrm{~mm} \times 12 \mathrm{~mm}$ samples.

We also prepared nanometer-sized powders of thermoelectric materials using mechanical means (via Ball Milling) and developed processes to consolidate and compact them to fabricate bulk nano-composite structures. We used a $6 \mathrm{lb}$ capacity, double barrel ball milling machine where material was crushed to fine powders in neoprene containers which use Zirconia Balls as grinding media which can provide reasonably fine (submicron to $20 \mathrm{~nm}$ ) powders without contamination. We have used this to produce nano powders of bismuth (Bi) doped lead telluride ( $\mathrm{PbTe}$ ) and thalium (Th) doped $\mathrm{PbTe}$. Hydrostatic pressing of n-type and p-type nanopowders in punch and dies of various dimensions was used to make disks. Fabricated in this way, the material had an immense number of internal grain boundary interfaces. The result of having those interfaces is that the thermal conductivity is decreased while the electrical properties appeared to be largely unaffected, so the reported $\mathrm{Z}$ was improved by 50\%. Table I below shows the results from this work and compares the properties of the single crystal material and the nano material. Our previous results for $\mathrm{Sb}_{2} \mathrm{Te}_{3}: \mathrm{Ag}$ are included for comparison.. 
Table I: Comparison of single crystal and nano material properties.

\begin{tabular}{|l|c|c|c|c|}
\hline \multicolumn{1}{|c|}{ Materials } & \multicolumn{2}{c|}{ PbTe:Bi } & \multicolumn{2}{c|}{$\mathrm{Sb}_{2} \mathrm{Te}_{3}: \mathrm{Ag}$} \\
\hline & Single & Nano & Single & Nano \\
\hline Seebeck Coefficient $[\mu \mathrm{V} / \mathrm{K}]$ & 96 & 499.23 & 126 & 125 \\
\hline Carrier Concentration $\left[\mathrm{cm}^{-3}\right]$ & $1.1 \times 10^{19}$ & $7.45 \times 10^{16}$ & $6.1 \times 10^{19}$ & $1.22 \times 10^{20}$ \\
\hline Mobility $\left[\mathrm{cm}^{2} / \mathrm{V}-\mathrm{sec}\right]$ & 1075 & 38.9 & 185 & 12.4 \\
\hline Resistivity $[\mathrm{mOhms} / \mathrm{cm}]$ & 0.529 & 2170 & 0.6 & 4.13 \\
\hline
\end{tabular}

Compared to the equivalent single-crystal, the carrier concentration dropped. That drop, along with the grain boundary scattering of electrons causes an overall increase of the electrical resistivity. These measurements point to the need for higher pressure, and/or higher annealing temperature during densification. The expectation is that with higher pressure and/or temperature, the carrier mobility will continue to increase and the carrier concentration will remain as high as desired, roughly $1.0 \times 10^{19} / \mathrm{cm}^{-3}$. The expected result is a further reduced electrical resistivity. We still need to optimize our nanostructured bulk materials before starting to build power generation devices. Meanwhile we have used our single crystalline materials and built a quad device for power generation.

\section{RESULTS AND DISCUSSION}

To test our materials, we have developed a new device fabrication technology, the"flip-chip assembly" that is based on bulk materials technology. The flip-chip assembly is a piece-wise assembly technique that lends itself nicely to a segmented, tandem-mosaic arrangement of devices electrically connected in series or parallel to reach the desired activearea. It would be straightforward to connect small TE device segments in tandem with series or parallel connections to reach a desired active area. There is a trade-off, however, between open-circuit voltage, short-circuit current, and the number of series and parallel connections. The open-circuit voltage will increase linearly with the number of seriesconnected modules while the short circuit current remains roughly constant. For parallel-connected modules, the opencircuit voltage remains roughly the same while the short-circuit current increases with the number of devices. The number of series and parallel connections can be easily designed and engineered to supply any standard voltage need (e.g., $120 \mathrm{~V}, 240 \mathrm{~V}$ ), and we note that there are approaches using MEMS switching to have a continuously variable open-circuit voltage power supply for applications where a good impedance match to any desired load can be obtained.

In order to achieve, ultimately, kilowatt power level generation, the fabrication proceeds by preparing each component in parallel to improve ease of assembly and yield. The components are shown in Figure 1 at the various stages of assembly. Once in position, the metallurgical junctions were formed by application of pressure and heat. Fabricated in this way, the obtained device shown in Figure $1 \mathrm{C}$ is mechanically rugged and fully operational. The initial characteristics of the device were determined immediately after assembly. The device's total electrical resistance was experimentally found to be $0.432 \Omega$ and remained stable throughout thermal testing. The resistivity of the oxygen-free copper is $1.7 \times 10^{-}$ ${ }^{6} \Omega$-cm, so the contribution to the total device resistance from the sum of the resistance from all six copper junctions is more than almost five orders of magnitude less than that from the semiconductor materials, so the dominant contributions would be only from the semiconductors, and potentially from the average contact resistance, $R_{c}$. 


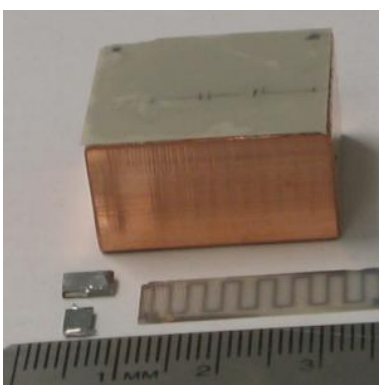

(a)

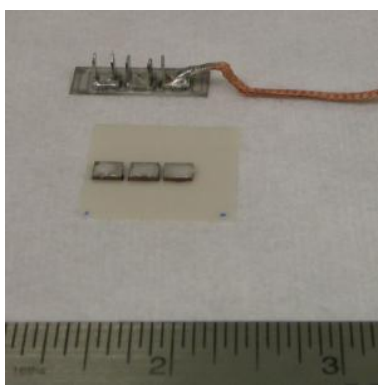

(b)

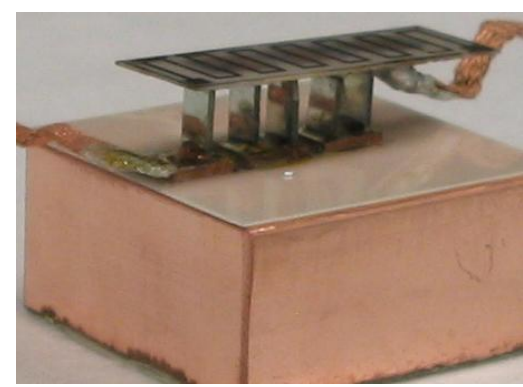

(c)

Figure 1. (a) Break-out view of the individual components hot-and cold-junctions, (b) view of the separate, and (c) the final device after flip-chip assembly.

The theoretical resistance of the device $\left(\mathrm{R}_{\mathrm{d}}\right)$ was calculated using the equation

$$
R_{d}=\sum_{j}\left(R_{c}\right)_{j}+\sum_{i} \rho_{i}\left(\frac{1_{i}}{A_{i}}\right)
$$

where $\rho$ was the measured resistivity values from independent Hall effect measurements listed, A is the cross-sectional area and 1 is the length for each of the individual " $\mathrm{i}$ " components along with each of the" $\mathrm{j}$ " contacts according to. $\mathrm{R}_{\mathrm{d}}$ for this device is $0.432 \Omega$, which is consistent with the experimental value. Because the theoretical and experimental resistance values are consistent to three significant figures, $R_{c}$ must be $10^{-5} \Omega-\mathrm{cm}^{2}$ or lower. The agreement between experimental and calculated $R_{d}$ is likely fortuitous, in part, because of the relative uncertainty from the geometry measurement, believed to be about $2 \%$. However, the fact that the measured and predicted values are obtained as the average of $\mathrm{j}=$ ten separate contacts highlights the fact that each contact must have excellent integrity and that the assembly technique is highly effective, robust, and practical

For higher temperature tests, the device was mounted in a vacuum system to prevent oxidation of the materials and reduce error from convective heat flow. To test the device for power generation, the hot-junction was heated radiatively by a $30 \mathrm{~W}$ "HotWatt" electrical power resistor. The resistor was not in contact with the TE device so as to minimize thermal mass. The output power was measured from the two braided copper terminals shown in Figure 3C. To assure that the device had reached a steady-state temperature difference, the range of temperature difference was slowly scanned from $\Delta \mathrm{T}=0$ to $100 \mathrm{~K}$. The power density is shown as a function of temperature difference in Figure 2 .

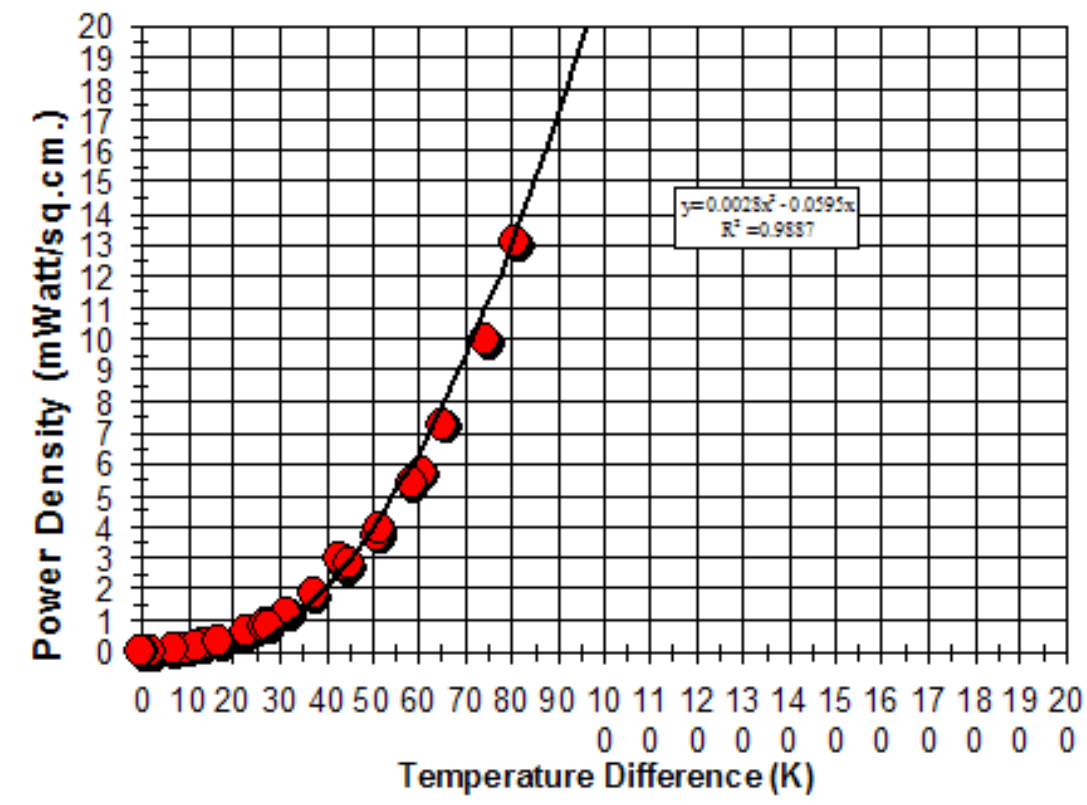

Figure 2. Power density $\left(\mathrm{mW} / \mathrm{cm}^{2}\right)$ vs Temperature Difference $[\mathrm{K}]$.

The absolute upper thermodynamic limit for power is set by the Carnot efficiency, which is set by the upper and lower bounding temperatures. Over a small temperature difference, $\Delta \mathrm{T} \sim 2 \mathrm{~K}$, Carnot efficiency would be about $0.6 \%$. For larger $\Delta \mathrm{T}$, however, the Carnot efficiency becomes greatly improved to the point where it is on the order of $25 \%$ for temperature differences greater than $100 \mathrm{~K}$. Accordingly, the TE efficiency is 
greatly improved. At realistic $\Delta \mathrm{T}(\sim 4-500 \mathrm{~K})$, power densities $\sim 0.5$ to $1.0 \mathrm{~W} / \mathrm{cm}^{2}$ would be obtained. Next, a Quad device was built and tested. A photograph of the device is shown in Fig. 3 and the results for the voltage and power vs temperature difference are shown in Fig. 4.

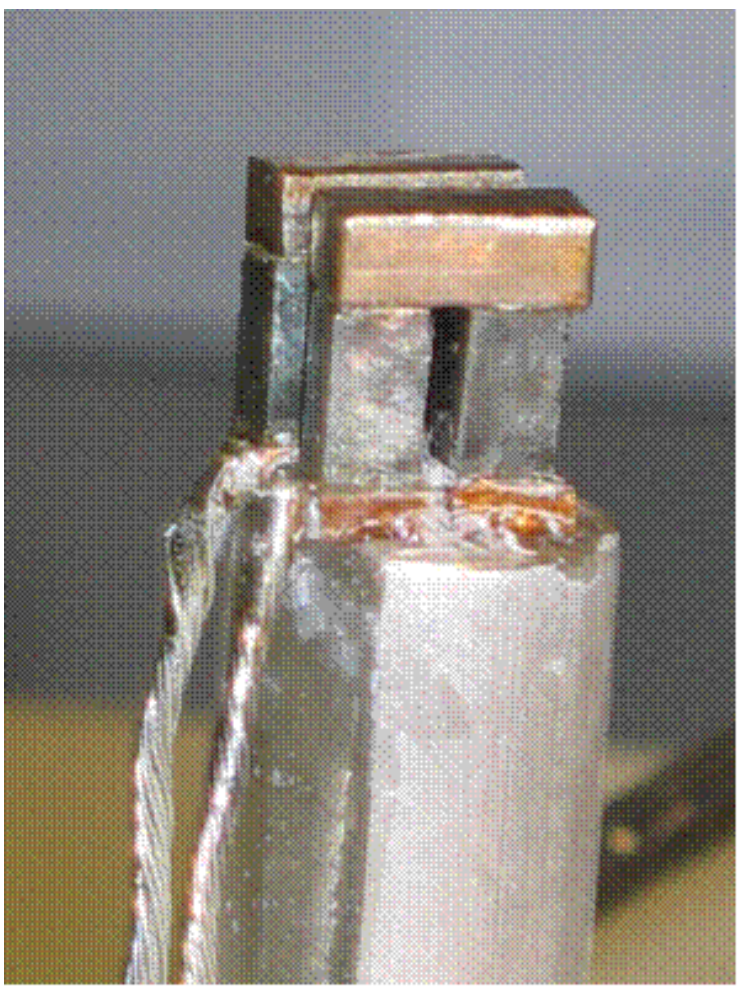

Fig. 3. Photograph of Quad Device

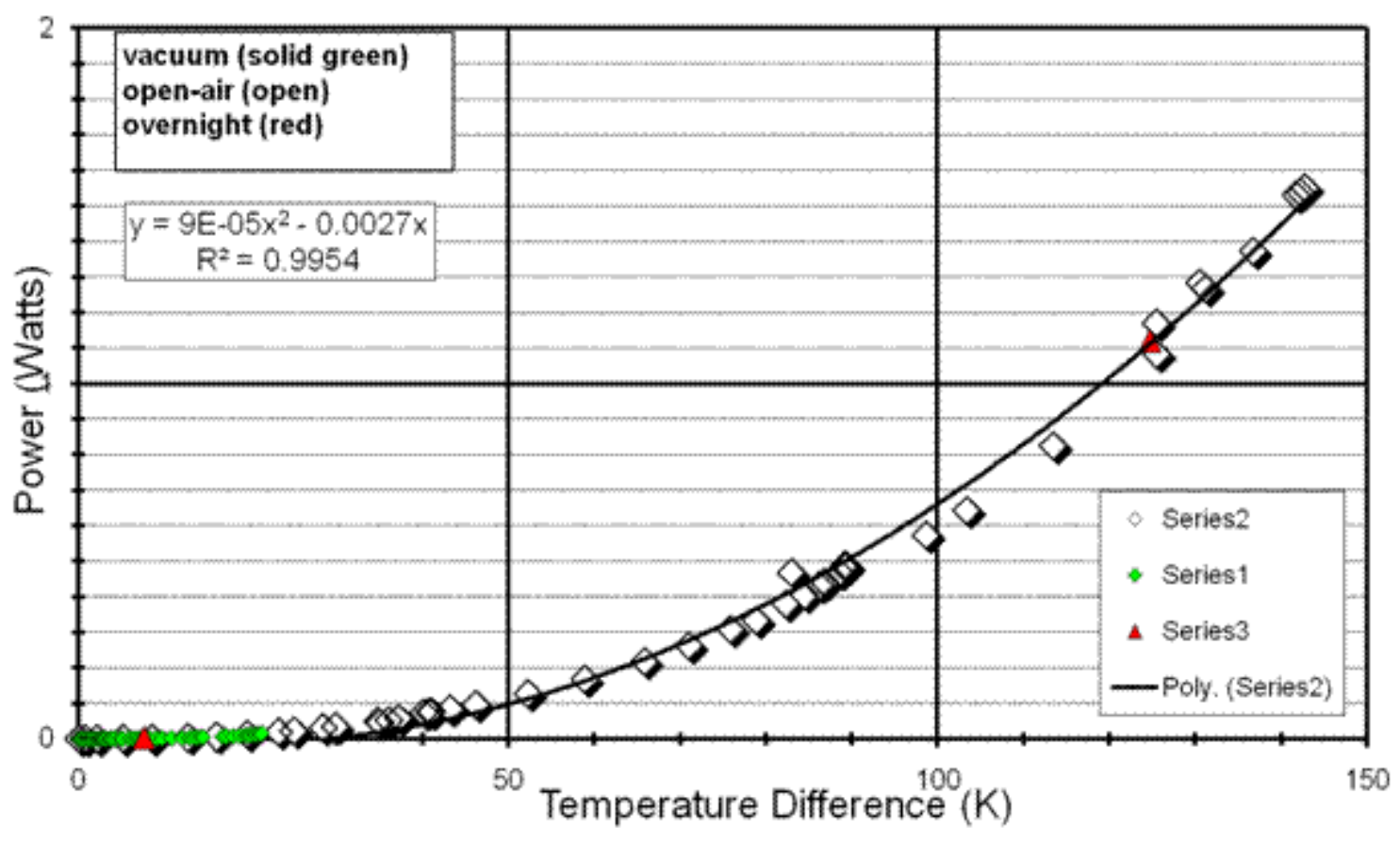

Fig. 4. Power vs temperature difference for Quad device in vacuum and open air. 
Thus, we demonstrated very low resistance contacts and good power generation consistent with state-of-the-art soldering/hand assembly with the novel flip-chip device assembly. This new fabrication can lead to highly-integrated, smaller scale devices, such as massive row-by-column TE arrays ( $10^{6} \mathrm{TE}$ devices per array), and can be used to fabricate larger scale devices where modules may be integrated onto a curved surface.

\section{CONCLUSIONS}

We produced $\mathrm{n}$ type $\mathrm{PbTe}$ by doping it with $\mathrm{Bi}$ and $\mathrm{p}$-type $\mathrm{PbTe}$ by doping it with thallium. We successfully grew single crystals of thallium doped doped and bismuth doped PbTe via the Bridgman technique. The thalium doped crystal provided p-type material and $\mathrm{Bi}$ doped $\mathrm{PbTe}$ gives n-type material for the thermoelectric power generators. We also prepared nanometer-sized powders of thermoelectric materials using mechanical means and developed processes to consolidate and compact them to fabricate bulk nano-composite structures. We are currently optimizing our processing techniques to produce device grade bulk nano-composites.

We have developed a new device fabrication technology, the "flip-chip assembly", that is based on bulk materials technology. The flip-chip assembly is a piece-wise assembly technique that lends itself nicely to a segmented, tandemmosaic arrangement of devices electrically connected in series or parallel to reach the desired active-area. The new "flipchip" module assembly technique is scalable, modular, and results in a low value of contact resistivity $\left(\leq 10^{5} \Omega-\mathrm{cm}^{2}\right)$. It can be used to leverage new advances in thin-film and nanostructured materials for the fabrication of new miniature thermoelectric devices. It may also enable monolithic integration of large devices or tandem arrays of devices on flexible or curved surfaces. Under mild testing, a power of $22 \mathrm{mWatt} / \mathrm{cm}^{2}$ was obtained from small $(<100 \mathrm{~K})$ temperature differences. At higher, more realistic temperature differences, $\sim 500 \mathrm{~K}$, where the efficiency of these materials greatly improves, this power density would scale to between 0.5 and $1 \mathrm{Watt} / \mathrm{cm}^{2}$. These results highlight the excellent potential for the generation and scavenging of electrical power of practical and usable magnitude for remote military using TE technology.

\section{REFERENCES}

[1] A. Ioffee, Semiconductor Thermoelements and Thermoelectric Cooling, London, Infosearch, ltd., p. 40 (1957).

[2] R. Venkatasubramanian, E. Siivola, T. Colpitts and B. O'Quinn, "Thin-film thermoelectric devices with high roomtemperature figures of merit", Nature Vol. 413, 597-602 (2001).

[3] T. C. Harman, P. J. Taylor, M. P. Walsh, B. E. LaForge, "Quantum Dot Superlattice Thermoelectric Materials and Devices", Science Vol. 297. no. 5590, pp. 2229.(2002).

[4] T. Hogan, A. Downey, J. Short, J. D’Angelo, C. Wu, E. Quarez, J. Androulakis, P. Poudeu, J. Sootsman, D. Chung, M. Kanatzidis, S.D. Mahanti, E. Timm, H. Schock, F. Ren, J. Johnson and E.. Case, "Nanostructured Thermoelectric Materials and High-Efficiency Power-Generation Modules", Journ. of Elect. Mat., Vol. 36, Number 7, p. 704 (2007).

[5] D. M. Rowe, V. Shukla, N. Savvides, Nature V. 290, No. 5809, p. 765, (1981).

[6]. http://www.spectrum.ieee.org/mar08/6081. (2008). 